\title{
Galactic evolution of Copper in the light of NLTE computations
}

\author{
S. Andrievsky ${ }^{1,2} \dagger$, P. Bonifacio ${ }^{2}$, E. Caffau ${ }^{2}$, S. Korotin, ${ }^{1,3}$, M. Spite ${ }^{2}$, F. Spite ${ }^{2}$, \\ L. Sbordone ${ }^{4}$ and A.V. Zhukova ${ }^{3}$ \\ ${ }^{1}$ Department of Astronomy and Astronomical Observatory, Odessa National University, Isaac Newton Institute of Chile, Odessa Branch, \\ Shevchenko Park, 65014, Odessa, Ukraine \\ ${ }^{2}$ GEPI, Observatoire de Paris, PSL Research University, CNRS, Place Jules Janssen, 92195 Meudon, France \\ ${ }^{3}$ Crimean Astrophysical Observatory, Nauchny 298409, Republic of Crimea \\ ${ }^{4}$ European Southern Observatory, Alonso de Cordova 310\%, Vitacura, Santiago, Chile
}

Accepted XXX. Received YYY; in original form ZZZ

\begin{abstract}
We have developed a model atom for $\mathrm{Cu}$ with which we perform statistical equilibrium computations that allow us to compute the line formation of $\mathrm{Cu}$ I lines in stellar atmospheres without assuming Local Thermodynamic Equilibrium (LTE). We validate this model atom by reproducing the observed line profiles of the Sun, Procyon and eleven metal-poor stars. Our sample of stars includes both dwarfs and giants. Over a wide range of stellar parameters we obtain excellent agreement among different $\mathrm{Cu}$ I lines. The eleven metal-poor stars have iron abundances in the range $-4.2 \leq[\mathrm{Fe} / \mathrm{H}] \leq$ -1.4 , the weighted mean of the $[\mathrm{Cu} / \mathrm{Fe}]$ ratios is $-0.22 \mathrm{dex}$, with a scatter of $-0.15 \mathrm{dex}$. This is very different from the results from LTE analysis (the difference between NLTE and LTE abundances reaches 1 dex) and in spite of the small size of our sample it prompts for a revision of the Galactic evolution of $\mathrm{Cu}$.
\end{abstract}

Key words: radiative transfer - line: formation - line: profiles - stars: atmospheres - stars: abundances - Galaxy: evolution

\section{INTRODUCTION}

Copper is an odd element that can be formed through several nucleosynthetic processes (Bisterzo et al. 2004), the relevant importance of the various processes is debatable. Up to now there has been a general consensus that, observationally, the $[\mathrm{Cu} / \mathrm{Fe}]$ ratio decreases with decreasing metallicity (e.g. Cohen 1980; Sneden et al. 1991; Mishenina et al. 2002; Simmerer et al. 2003; Bihain et al. 2004, see Bonifacio et al. 2010 for a concise summary of the observations). At very low metallicity the only lines that are strong enough to be measured on ground-based spectra are the UV Cu I resonant doublet at $3247 \AA$ and $3273 \AA$. Bihain et al. (2004) and Bonifacio et al. (2010) pointed out that, when computed under the assumptions of Local Thermodynamic Equilibrium (LTE), these lines are discrepant with the optical lines. Roederer et al. (2014) could measure vacuum UV Cu II lines in two metal-poor stars, on spectra taken with the GHRS

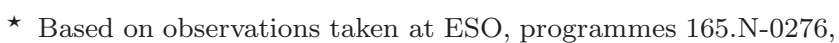
65.L-0165，65.L-0507，266.D-5655，66.B-0378，67.D-0439，68.D0546, 70.D-0009, 71.B-0529, 072.B-0585, 078.D-0643 and HST programme GO-14161

$\dagger$ E-mail: andrievskii@ukr.net and STIS spectrographs on the Hubble Space Telescope and found that they were strongly discrepant with the UV Cu I resonant lines. Collectively these observations prompt to consider the line formation of $\mathrm{Cu}$ I lines relaxing the hypothesis of Local Thermodynic Equilibrium. Such an investigation was conducted by Shi et al. (2014), however they only considered the optical lines and not the UV resonant doublet. The model atom developed by Shi et al. (2014) was used by Yan et al. (2015) to investigate a sample of stars covering the range in metallicity -1.9 to -0.2 . In this investigation they confirmed the decrease of the $[\mathrm{Cu} / \mathrm{Fe}]$ ratios with decreasing metallicity, albeit with higher $[\mathrm{Cu} / \mathrm{Fe}]$ ratios at the lowest metallicity, with respect to the previous LTE analysis. In a subsequent investigation Yan et al. (2016) claimed that their NLTE analysis confirmed also the differences in $\mathrm{Cu}$ abundances between the high- $\alpha$ and low- $\alpha$ populations found by Nissen \& Schuster (2011) in the metallicity range -1.5 to -0.4 .

The main purpose of our investigation is to see whether a NLTE approach is capable of reconciling the $\mathrm{Cu}$ abundances derived from the UV resonant doublet and the optical lines in metal-poor stars. For this purpose we developed a $\mathrm{Cu}$ model atom, and after a first validation on the spectra of the Sun and Procyon we applied it to a small sample of 


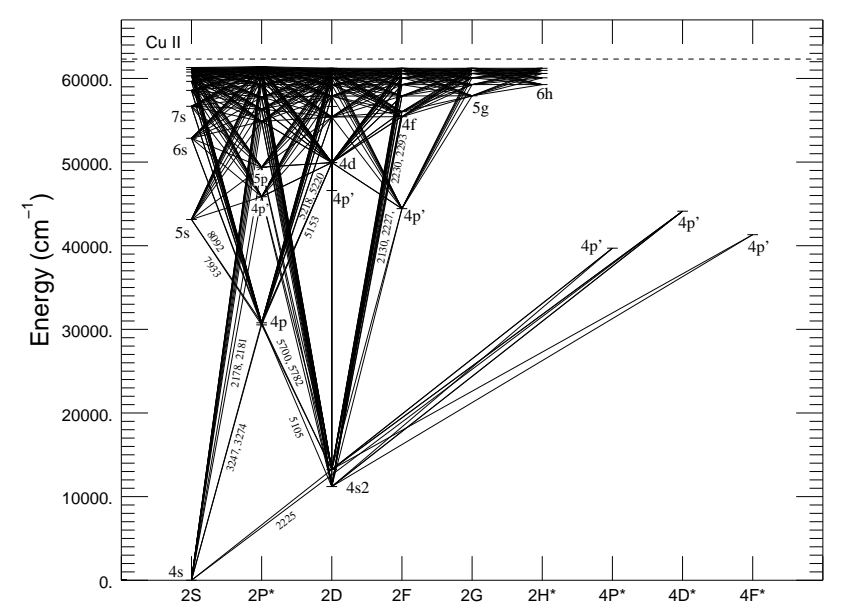

Figure 1. The Grotrian diagram of our $\mathrm{Cu}$ model atom.

metal-poor stars covering the range of -4.2 to -1.4 in $[\mathrm{Fe} / \mathrm{H}]$. When possible we complemented the ground-based spectra with vacuum UV spectra observed with Hubble Space Telescope.

\section{COPPER MODEL ATOM}

To build a model atom of copper we used 130 levels (116 levels of $\mathrm{Cu}$ I and 14 levels of $\mathrm{Cu}$ II). Parameters of atomic levels were taken from Liu et al. (2014), Sugar \& Musgrove (1990) and the NIST database. Each LS multiplet was considered as a single term. The fine structure was taken into account for the following levels: $4 \mathrm{~s}^{2} 2 \mathrm{D}, 4 \mathrm{p}^{2} \mathrm{P}^{\mathrm{o}}$ and $4 \mathrm{~d} 2 \mathrm{D}$, that are tightly coupled with the most important transitions in the $\mathrm{Cu}$ I atom (it should be noted that the fine splitting in this case is very important: for instance the splitting of $4 \mathrm{~s}^{2} 2 \mathrm{D}$ is larger than $0.25 \mathrm{eV}$ ). The highest energy level in our $\mathrm{Cu}$ I model for which we accounted for the radiative and collisional transitions has a ionization potential $0.11 \mathrm{eV}$ that corresponds to an electronic temperature of about $1300 \mathrm{~K}$. Thus, we can conclude that our model describes the connection between excited levels and continuum quite accurately. The adopted Grotrian diagram of or $\mathrm{Cu}$ atomic model is shown in Fig.1.

We have considered radiative transitions between the first 59 levels of $\mathrm{Cu}$ I and ground level of $\mathrm{Cu}$ II. Populations of the other levels were taken into account only in the solution of the particle number equation. All 486 bound-bound transitions were considered in detail. For each transition we accounted for Stark and van der Waals effects, as well as for the influence of the microturbulent velocity. To adequately reproduce the radiative rates of the several significant UV transitions from $4 \mathrm{~s} 2 \mathrm{~S}$ and $4 \mathrm{~s}^{2} 2 \mathrm{D}$ levels we calculated the corresponding profiles for 100 to 150 frequency points. For the rest of the line profiles we used only 30 frequency points. Photoionization cross-sections were taken from Liu et al. (2014). For all the bound-bound transitions the oscillator strengths were compared to those from the NIST database, and if $\log$ gf values were absent there, we used the values provided by theoretical calculations of Liu et al. (2014).

Collisional ionization rates were calculated basing on the Seaton's formula (Seaton 1962) with threshold photoion- ization cross-section value from Liu et al. (2014). Collisional excitations by electrons were calculated with the help of van Regemorter's formula (van Regemorter 1962). Collisional rates for the forbidden transitions can be found with Allen's formula (Allen 1973) with an effective collisional strength of 1 . For the 30 transitions between the low excited levels we used the collisional rates calculated by M. O'Mullane, and made available through the Atomic Data and Analysis Structure (ADAS, Summers 2004). Inelastic collisions with hydrogen atoms were described with the Drawin's formula (Drawin 1968, 1969) adapted for astrophysical use by Steenbock \& Holweger (1984).

Atomic level populations were determined using the MULTI code of Carlsson (1986) with modifications as given in Korotin et al. (1999). MULTI calculates the line profile for each line considered in detail. The line profile computed assuming either LTE or NLTE depends upon many parameters: the effective temperature of the model, the surface gravity, the microturbulent velocity, and the line damping as well as the populations in the appropriate levels. Proper comparison of observed and computed profiles in many cases requires a multielement synthesis to take into account possible blending lines of other species. For this process, we fold the NLTE (MULTI) calculations, specifically the departure coefficients, into the LTE synthetic spectrum code SYNTHV (Tsymbal 1996) that enables us to calculate the NLTE source function for copper lines. These calculations included all spectral lines from the VALD database (Ryabchikova et al. 2015) in a region of interest. The LTE approach was applied for lines other than the $\mathrm{Cu}$ I lines. Abundances of corresponding elements were adopted in accordance with the $[\mathrm{Fe} / \mathrm{H}]$ value for each star.

$\mathrm{Cu}$ I has several lines that are suitable for abundance determination in the visual range: $5105 \AA$, $5153 \AA$, $5218 \AA, 5220 \AA, 5700 \AA, 5782 \AA$ (oscillator strengths are from Kock \& Richter 1968), and in IR range: $7933 \AA$ А, $8092 \AA$ (oscillator strengths are from Carlsson 1988). Two resonance UV lines $3247 \AA$ and $3273 \AA$ are too strong in the solarmetallicity stars. Nevertheless, these lines can be important as a copper abundance indicator in the metal-poor stars (Bihain et al. 2004; Bonifacio et al. 2010). The log gf value for $5782 \AA$ line is $-1.78( \pm 12 \%)$ in Kock \& Richter (1968), and -1.91 in Liu et al. (2014). From our solar spectrum analysis we obtained $\log \mathrm{gf}=-1.83$. All the line parameters we used are given in Table 2. We adopted the following isotopic ratio ${ }^{63} \mathrm{Cu}$ to ${ }^{65} \mathrm{Cu}=0.69 / 0.31$ (recommended by Grevesse et al. 2015). For most of the lines we used the hyperfine structure $(h f s)$ component ratios and wavelength shifts from Shi et al. (2014). As a control, we also performed the synthesis of the $\mathrm{Cu}$ I line profiles in the solar spectrum using the corresponding data from Kurucz's list (Kurucz 2014, 2011, 2005b). We did not obtain any significant differences. For the better reproduction of the profiles of the resonance doublet $3247 \AA$, and $3273 \AA$ and the high excitation line $5105 \AA$ line in the solar spectrum we used the $h f_{s}$ component shifts from the magnetic dipole splitting constants $\mathrm{A}(\mathrm{J})$ and electric quadrupole splitting constants $\mathrm{B}(\mathrm{J})$ from Gerstenberger et al. (1979) and Hermann et al. (1993). Components with wavelength differences less than $0.001 \AA$ were combined into one component. Results are given in Table 2 .

Since the strong $\mathrm{Cu}$ I lines are formed in the upper at- 
Table 1. Programme stars and adopted stellar parameters.

\begin{tabular}{|c|c|c|c|c|c|c|}
\hline Name & $\begin{array}{c}T_{\text {eff }} \\
\mathrm{K}\end{array}$ & $\begin{array}{c}\log g \\
\mathrm{~g} \text { in cgs }\end{array}$ & $\begin{array}{c}\xi \\
\mathrm{km} \mathrm{s}^{-1}\end{array}$ & $\begin{array}{c}{[\mathrm{Fe} / \mathrm{H}]} \\
\operatorname{dex}\end{array}$ & & $\begin{array}{c}\mathrm{A}(\mathrm{Cu}) \\
\operatorname{dex}\end{array}$ \\
\hline HD 111721 & 5095 & 2.64 & 1.4 & -1.40 & 1 & $2.87 \pm 0.05$ \\
\hline HD 94028 & 5970 & 4.33 & 1.3 & -1.47 & 2 & $2.60 \pm 0.03$ \\
\hline Cl* NGC 6752 YGN 30 & 4943 & 2.42 & 1.3 & -1.62 & 3 & $2.35 \pm 0.10$ \\
\hline HD 9051 & 4925 & 1.95 & 1.8 & -1.78 & 1 & $2.41 \pm 0.04$ \\
\hline HD 84937 & 6300 & 4.00 & 1.3 & -2.25 & 4 & $1.80 \pm 0.02$ \\
\hline HD 128279 & 5040 & 2.22 & 1.4 & -2.45 & 1 & $1.50 \pm 0.06$ \\
\hline HD 140283 & 5750 & 3.70 & 1.4 & -2.59 & 5 & $1.40 \pm 0.02$ \\
\hline HD 122563 & 4600 & 1.10 & 2.0 & -2.82 & 6 & $1.18 \pm 0.03$ \\
\hline CS 31082-001 & 4825 & 1.50 & 1.8 & -2.91 & 6 & $1.35 \pm 0.10$ \\
\hline HD 200654 & 5007 & 2.21 & 1.0 & -3.16 & 1 & $0.91 \pm 0.05$ \\
\hline $\mathrm{CD}-38^{\circ} 245$ & 4800 & 1.50 & 2.2 & -4.19 & 6 & $0.04 \pm 0.10$ \\
\hline
\end{tabular}

References for the atmospheric parameters

1. This paper

2. Sitnova et al. (2015)

3. Yong et al. (2008)

4. Spite et al. (2017)

5. Siqueira-Mello et al. (2015)

6. Cayrel et al. (2004)

mosphere layers we applied the combination of the ATLAS solar model atmosphere of Castelli \& Kurucz (2003) with a chromosphere from the VAL-C model Vernazza et al. (1981) and with corresponding distribution of the microturbulent velocity. Observed line profiles were taken from the solar flux spectrum (Kurucz et al. 1984). All the line profiles were calculated assuming the $\mathrm{Cu}$ meteoritic abundance $(\mathrm{A}(\mathrm{Cu})=$ $\log (\mathrm{Cu} / \mathrm{H})+12=4.25$, Lodders et al. 2009). To reproduce the copper lines in the solar spectrum we used the collisional rates with atomic hydrogen atoms without any correcting factor. If one uses the value adopted by Shi et al. (2014), then the NLTE effects lead to a significant unbalance of the calculated line intensities and this implies an increase the copper abundance in solar atmosphere. We show the calculated and observed profiles of some lines in the solar spectrum in Fig. 2. All the investigated Cu I lines are weakened by the NLTE effects. Moreover, the line cores cannot be correctly described within the LTE approximation. As a further verification of our $\mathrm{Cu}$ I model atom we studied the spectra of Procyon. We obtained an excellent agreement between observed and calculated $\mathrm{Cu}$ I profiles adopting a copper abundance $\mathrm{A}(\mathrm{Cu})=4.30 \pm 0.03$. (see Fig. 3).

\section{COPPER ABUNDANCES IN STARS OF DIFFERENT METALLICITY}

In order to gain some insight in the Galactic evolution of $\mathrm{Cu}$ we applied our model atom and NLTE analysis to a set of stars for which we could find good quality visual and UV spectra in the ESO archive and which span a large range in metallicity. We requested that for each star the spectrum of the UV doublet be available, this is covered for all stars by observations obtained with UVES. For two stars (HD 84937 and HD 140283) we could also use HST STIS spectra, that gave us access to the vacuum ultra-violet. For HD 140283 we also made use of HARPS spectra. For HD 111721, HD 9051 and HD 128279 we determined the atmospheric parameters from the analysis of the visible spectra. We used the MyGIsFOS code (Sbordone et al. 2014) and the grids of synthetic spectra used by the MyGIsFOS code in the Gaia-ESO survey (see Smiljanic et al. 2014; Duffau et al. 2017). The temperatures were derived from the iron excitation equilibrium and the surface gravities from the iron ionisation equilibrium. For the other stars we adopted atmospheric parameters from the literature. The names, adopted stellar parameters and NLTE $\mathrm{Cu}$ abundances are listed in Table 1.

For each star we computed a 1D LTE model atmosphere using version 9 of the ATLAS code Kurucz (1993, 2005a) in the Linux version detailed by Sbordone et al. (2004); Sbordone (2005) and the Opacity Distribution Functions of (Castelli \& Kurucz 2003) with microturbulence of $1 \mathrm{~km} \mathrm{~s}^{-1}$.

For all of the programme stars we obtain a good consistency of the abundance implied by the different lines, as shown in Fig. 4 and 5. In particular for the metal-poor giants we always obtain the consistency between the UV doublet and the high excitation $5105 \AA$ line. In spectra of two program stars HD 9051 and HD 111721 the line $5218 \AA$ is also seen (Fig. 4). Its profile is well reproduced by our NLTE calculations. Accuracy of the profile fitting can be estimated, for instance, in Fig. 6 where we show LTE and NLTE profiles (two left panels), and NLTE profile variation with best copper abundance changed by \pm 0.3 dex (two right panels). Bonifacio et al. (2010) pointed out that this was never possible in LTE, even when using 3D hydrodynamical simulations. In Fig. 6 and 7 we show that the NLTE profiles reproduce well the observed profiles.

We consider as a success of our NLTE model atom the fact that for HD 84937 and HD 140283 we obtain consistency between the UV doublet $3242 \AA$ and $3273 \AA$ and the vacuum UV lines: $2165 \AA, 2178 \AA, 2181 \AA$, $2199 \AA, 2214 \AA$, $2225 \AA, 2227 \AA$, and $2230 \AA$. The $3273 \AA, 2165 \AA, 2178 \AA$, $2181 \AA$, and $2199 \AA$ lines in HD 84937 and HD 140283 are shown in Fig. 8. For these two stars, we also derived the copper abundance in LTE from four Cu II lines: $2112.100 \AA$, 2126.044 A, 2148.984 $\AA$ and 2247.003 A. Our copper model atom is not designed to compute the NLTE of the Cu II transitions. Since ionised copper is the dominant species in the atmospheres of our programme stars we expect deviations from LTE to be small. The parameters of the lines were taken from the VALD database (Ryabchikova et al. 2015). We obtained $\langle(\mathrm{Cu} / \mathrm{H})\rangle=1.71 \pm 0.07$ for $\mathrm{HD} 84937$ and $<(\mathrm{Cu} / \mathrm{H})>=1.31 \pm 0.08$ for HD 140283. We consider the agreement satisfactory, the difference with the NLTE-results for $\mathrm{Cu}$ I is less than 0.10 dex. We stress that for $\mathrm{Cu}$ I the difference between LTE and NLTE is of 0.70-0.80 dex.

As it was noted in Shi et al. (2014) and Yan et al. (2015, 2016) the deviations from LTE lead to the copper overionization already in the deep atmosphere layers. In Fig. 9 the distribution of departure coefficients $\left(b_{\mathrm{i}}=\frac{n_{\mathrm{i}_{\mathrm{NLTE}}}}{n_{\mathrm{i} \mathrm{LTE}}}\right)$ are shown for Procyon (the star with solar metallicity) and the metal deficient star HD140283. As one can see, the deviations from LTE start to become important at the optical depth of about 1. For the stars with solar metallicity the resonant doublet is practically not affected by the NLTE effects. These effects are gradually increasing as metallicity decreases, and reach large values $(-0.7$ dex for HD 84937 and -0.75 dex for HD 140283). Subordinate copper lines show stronger NLTE 

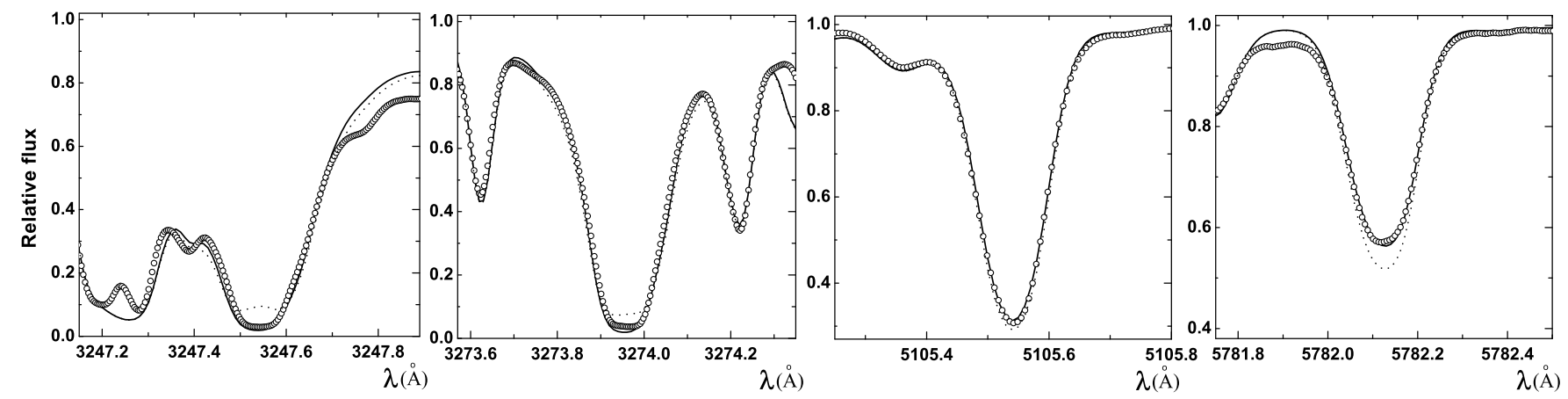

Figure 2. Several Cu I lines in the observed flux spectrum of the Sun (Kurucz et al. 1984), open circles, compared to our NLTE synthetic spectrum (solid) line and LTE synthetic spectrum (dotted line).
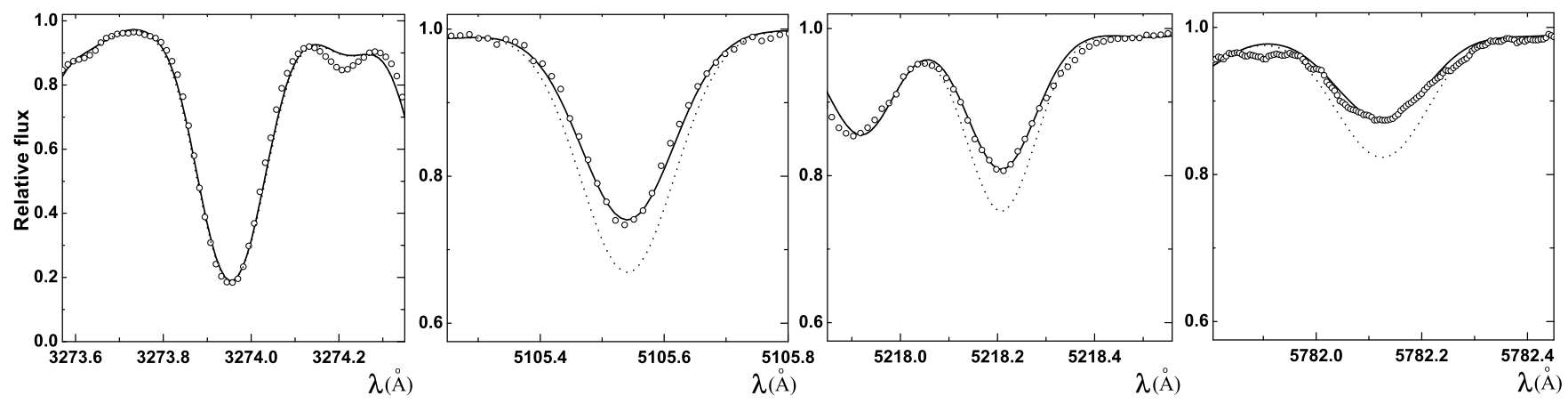

Figure 3. Several $\mathrm{Cu}$ I lines in the observed spectrum of the Procyon, open circles, compared to our NLTE synthetic spectrum (solid) line and LTE synthetic spectrum (dotted line).
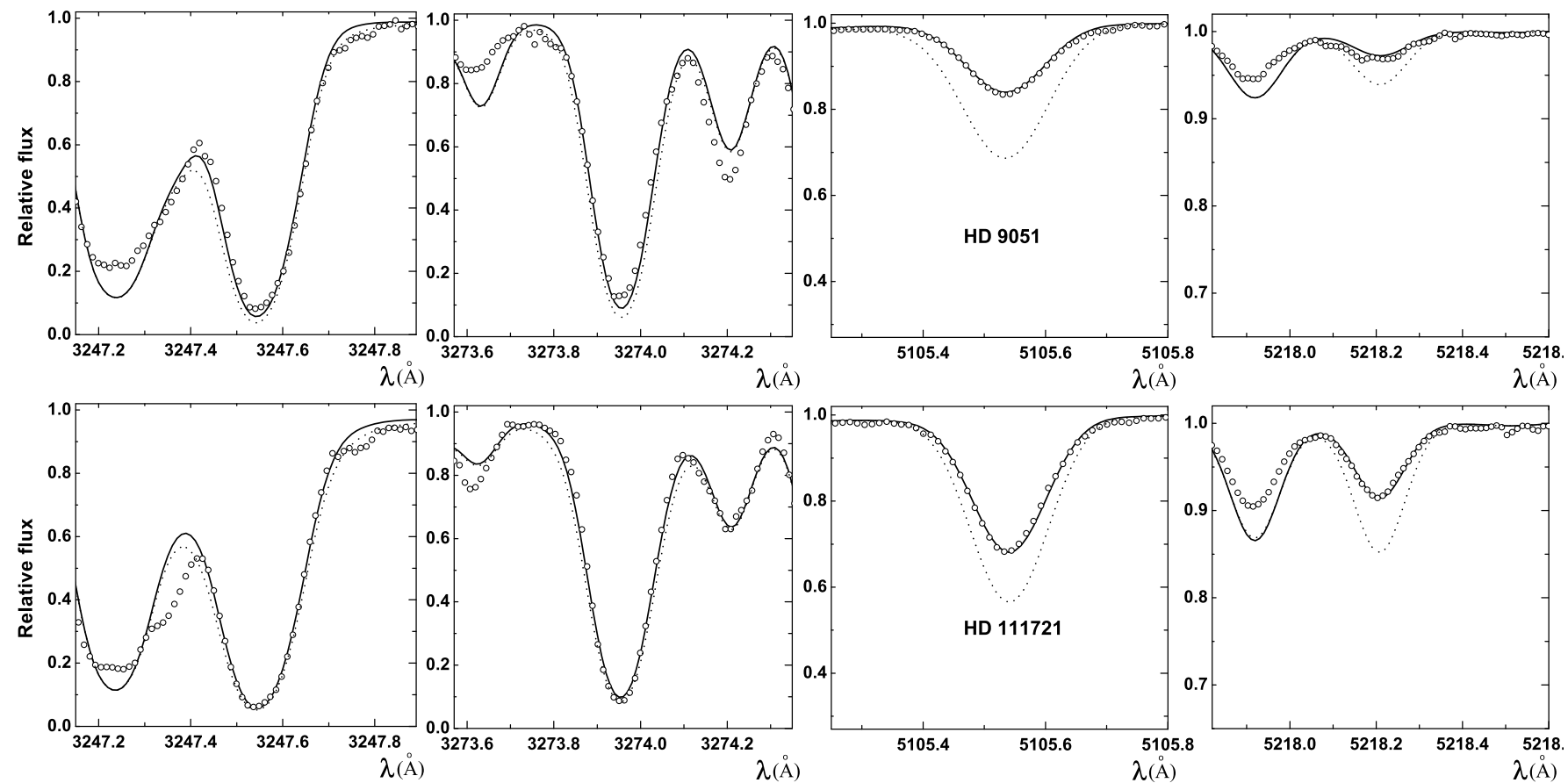

Figure 4. Cu I lines in the observed spectrum of the HD 9051 (upper panel), HD 11721 (lower panel), open circles, compared to our NLTE synthetic spectrum (solid) line and LTE synthetic spectrum (dotted line). 

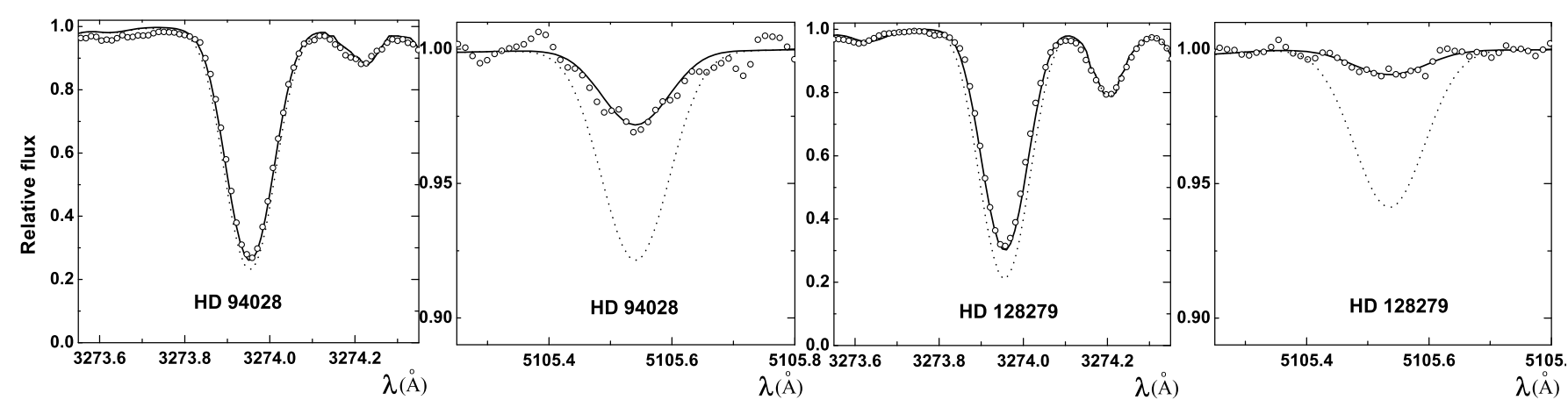

Figure 5. Cu I lines in the observed spectrum of the HD 94028 (two panels on the left) and HD 128279 (two panels on the right), open circles, compared to our NLTE synthetic spectrum (solid) line and LTE synthetic spectrum (dotted line).
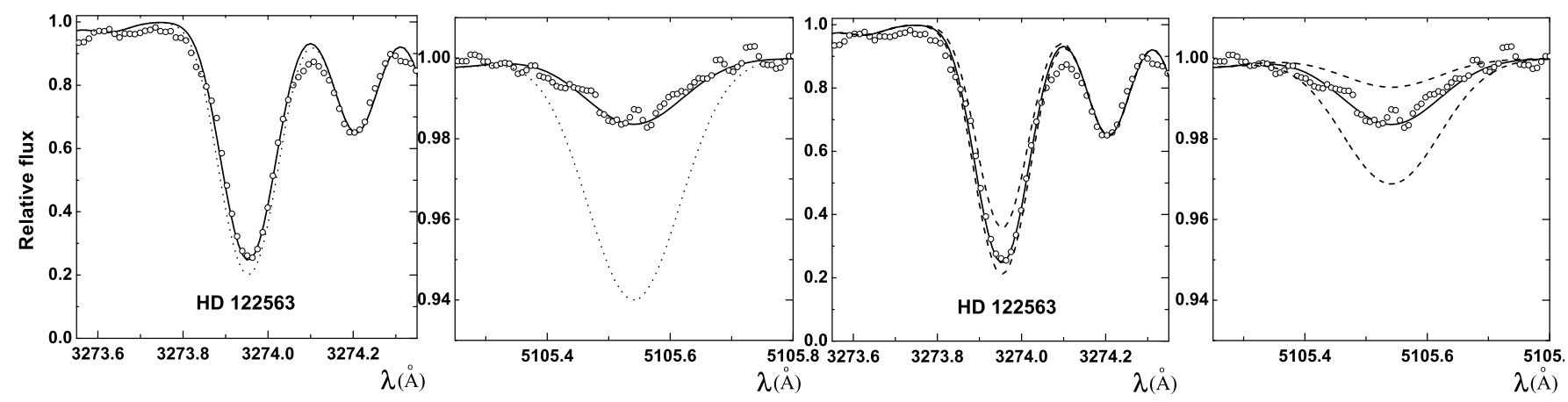

Figure 6. Cu I lines in the observed spectrum of the HD 122563, open circles, compared to our NLTE synthetic spectrum (solid) line and LTE synthetic spectrum (dotted line). The two panels on the left show the best fitting NLTE profiles, the two panels on the right show NLTE profiles computed with a $\mathrm{Cu}$ abundance of \pm 0.3 dex of the best fitting abundance.
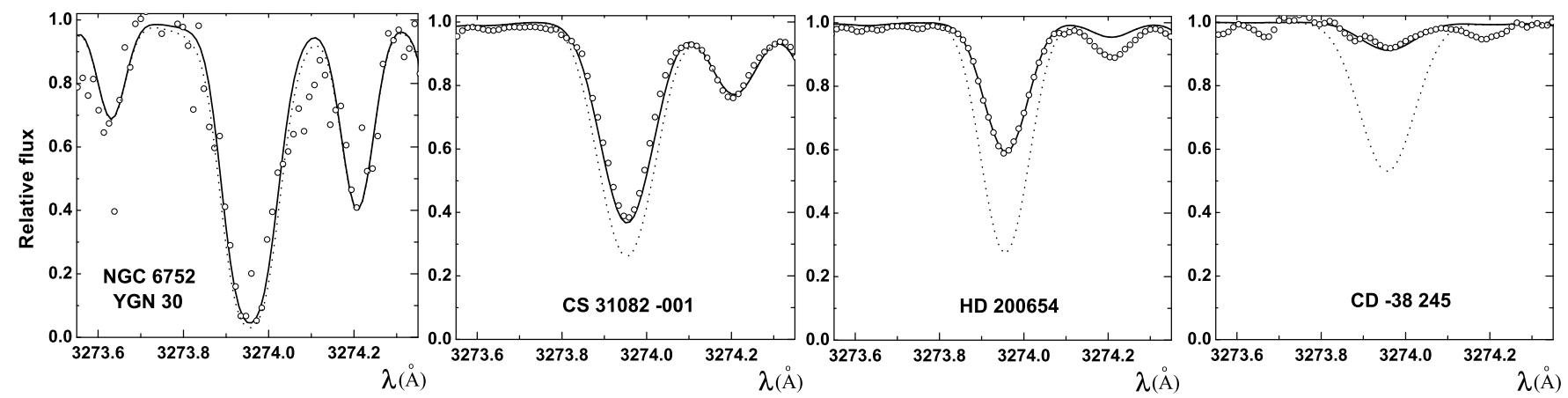

Figure 7. The CuI $3273 \AA$ A line in Cl* NGC 6752 YGN 30, CS 31082-001, HD 200654 and CD $-38^{\circ} 245$, open circles, compared to our NLTE synthetic spectrum (solid) line and LTE synthetic spectrum (dotted line).

effects even for the stars with solar metallicity, which are seen for example in Procyon.

As in the case of UV resonance doublet, the NLTE effects increase as metallicity decreases, but with different magnitude for different lines in some cases it can be as large as 1 dex. At the same time it should be noted that in the spectra of metal-poor stars subordinate lines become too weak to be accurately measured. For the stars with $T_{\text {eff }}$ about $6000 \mathrm{~K}$ and with $[\mathrm{Fe} / \mathrm{H}]$ of about -1.5 only the subordinate line at $5105 \AA$ is available for analysis. In the spectra of cooler stars, the line $5782 \AA$ can also be seen. These lines rapidly weaken with decreasing metallicity and completely disappear in the spectra of all stars at $[\mathrm{Fe} / \mathrm{H}]$ of about -2.5 . The NLTE corrections significantly depend on atmospheric parameters (like $T_{\text {eff }}, \log g, V_{\mathrm{t}},[\mathrm{Fe} / \mathrm{H}]$ ), as well as on the copper abundance itself. 

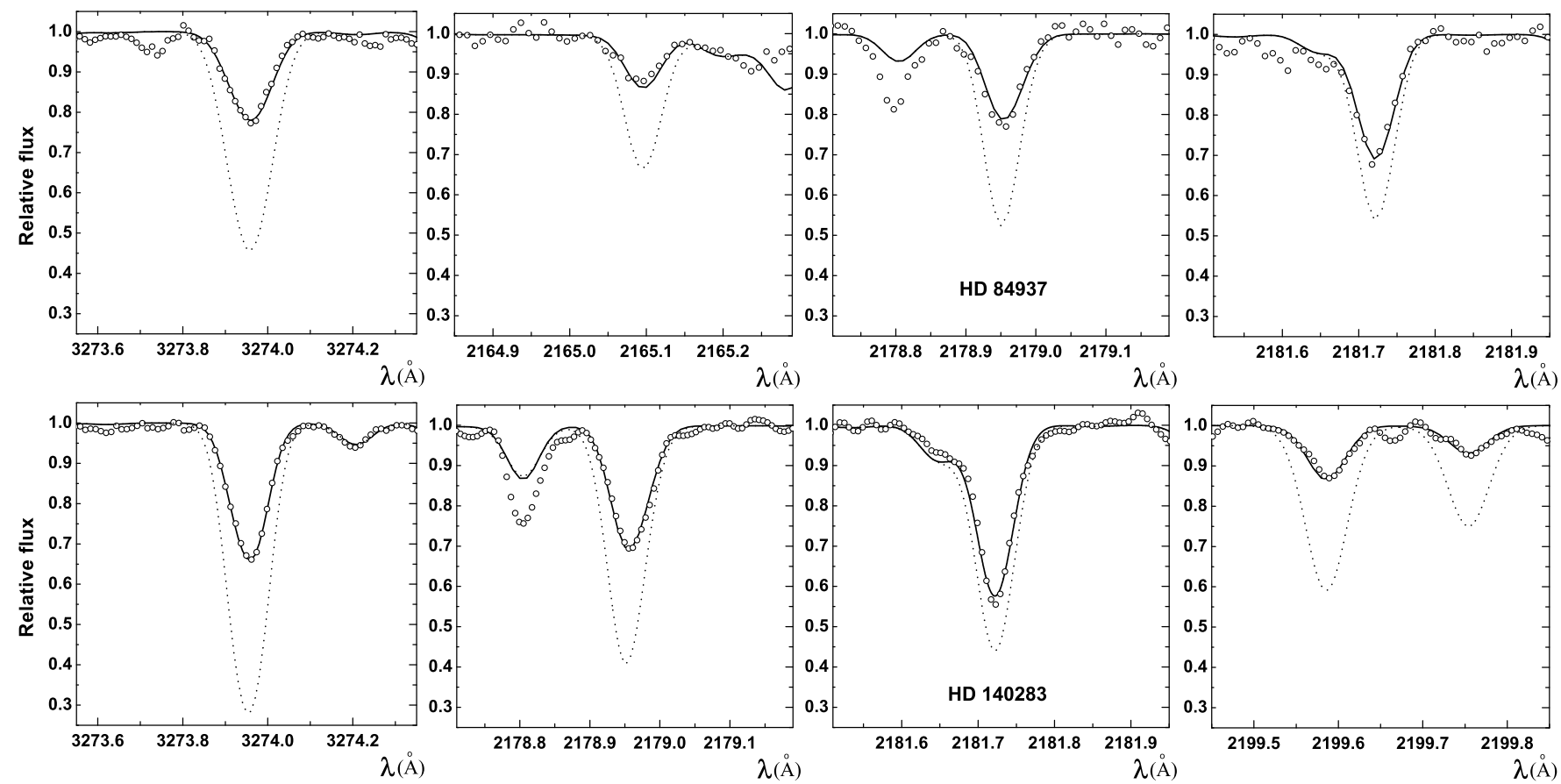

Figure 8. The CuI $3273 \AA$, $2165 \AA, 2178 \AA, 2181 \AA$, and $2199 \AA$ lines in the spectrum of the HD 84937 (upper panel) and HD 140283 (lower panel), open circles, compared to our NLTE synthetic spectrum (solid) line and LTE synthetic spectrum (dotted line).
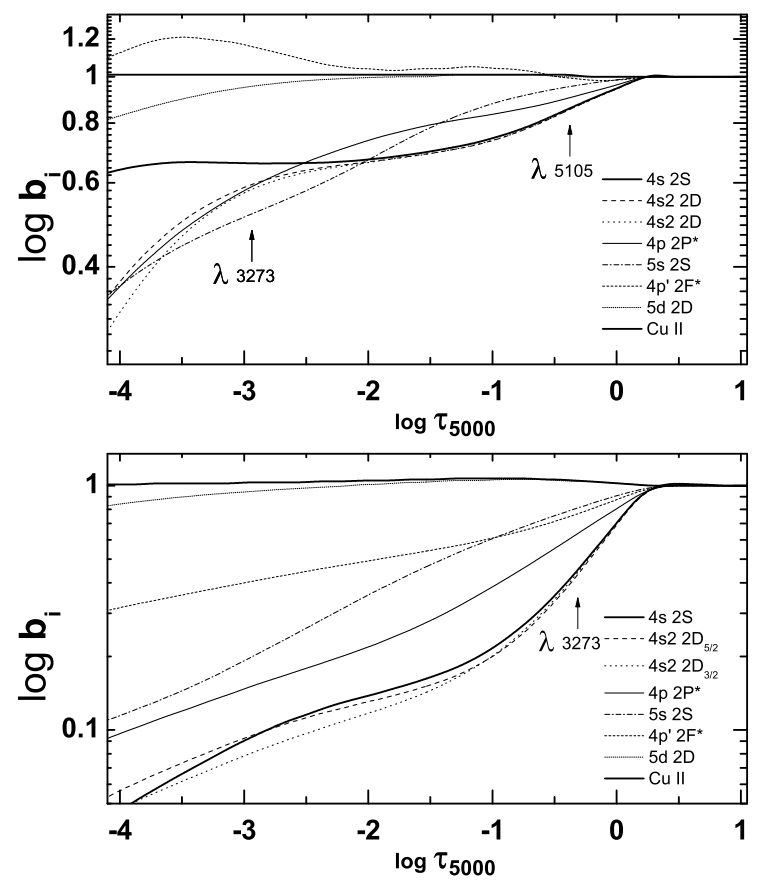

Figure 9. Computed departure coefficients as a function of optical depth in the atmosphere of Procyon (upper panel) and HD 140283 (lower panel).

\section{DISCUSSION}

Our sample of stars is very small and spans the metallicity range $-4.2 \leq[\mathrm{Fe} / \mathrm{H}] \leq-1.5$. We do not claim that we are in the best possible position to discuss the Galactic evolu- tion of copper, yet a few results are already noticeable. The fact that our NLTE analysis derives consistent abundances from different lines, including the strong vacuum UV lines, when available, allows us to conclude that the UV resonant doublet at $3247 \AA$ and $3273 \AA$ is a reliable indicator of the copper abundance. This is important because this doublet is the only one available to measure the copper abundance in low metallicity stars. We consider quite remarkable that we could measure the doublet in $\mathrm{CD}-38^{\circ} 245$, that is, to our knowldedge, the measurement of $\mathrm{Cu}$ at lowest metallicity so far achieved. We also checked the UVES spectra of HE 0107-5240 $([\mathrm{Fe} / \mathrm{H}]=-5.46$ Christlieb et al. 2004), but we could not detect any of the two lines. Based on the UVES spectra of $\mathrm{CD}-38^{\circ} 245$ the lines can be detected down to $[\mathrm{Fe} / \mathrm{H}]=-4.8$ in cool giants. Up to now the measurements of $\mathrm{Cu}$ at very low metallicity (Bihain et al. 2004; Lai et al. 2008; Cohen et al. 2008; Roederer et al. 2012, 2014) rely on the UV resonant lines. Our investigation shows that these measurements have to be revised to take into account the NLTE effects on these lines.

In Fig. 10 we show the run of $[\mathrm{Cu} / \mathrm{Fe}]$ as a function of $[\mathrm{Fe} / \mathrm{H}]$ for our programme stars. We also show the solar value, for reference. The main result that is apparent is that there are no very low $[\mathrm{Cu} / \mathrm{Fe}]$ values at variance with what happens in the LTE analysis. Our sample is more metal-poor than the sample of Yan et al. (2015), and we have only one star, HD 94028 in common. The adopted stellar parameters for this star are very close, yet we provide $[\mathrm{Cu} / \mathrm{Fe}]=-0.18 \pm 0.03$ while Yan et al. (2015) provide $-0.32 \pm 0.06$. Their abundance is based only on the $5105 \AA$ line while ours relies also on the $2 \mathrm{UV}$ resonance lines. From the $5105 \AA$ line we derive $[\mathrm{Cu} / \mathrm{Fe}]=-0.14$ and from the two resonance lines -0.22 . Our NLTE correction for the $5105 \AA$ 
Table 2. Atomic data for the lines $\mathrm{Cu}$ I.

\begin{tabular}{|c|c|c|c|c|c|c|c|}
\hline $\begin{array}{c}\lambda \\
(\AA)\end{array}$ & $\begin{array}{c}\log g f \\
h f s\end{array}$ & $\begin{array}{l}\log g f \\
\text { line }\end{array}$ & Ref & $\begin{array}{c}\lambda \\
(\AA)\end{array}$ & $\begin{array}{c}\log g f \\
h f s\end{array}$ & $\begin{array}{c}\log g f \\
\text { line }\end{array}$ & Ref \\
\hline 2165.096 & & -0.840 & 1 & 5700.143 & -4.298 & -2.58 & 3 \\
\hline 2178.949 & & -0.586 & 1 & 5700.159 & -4.094 & & \\
\hline 2181.722 & & -0.741 & 1 & 5700.164 & -4.696 & & \\
\hline 2199.586 & & 0.447 & 1 & 5700.166 & -4.298 & & \\
\hline 2199.754 & & 0.340 & 1 & 5700.173 & -3.948 & & \\
\hline 2214.583 & & 0.108 & 1 & 5700.188 & -3.744 & & \\
\hline 2225.705 & & -1.205 & 1 & 5700.193 & -4.346 & & \\
\hline 2227.776 & & 0.460 & 1 & 5700.194 & -4.152 & & \\
\hline 2230.086 & & 0.642 & 1 & 5700.195 & -3.948 & & \\
\hline \multirow[t]{2}{*}{2293.844} & & -0.115 & 1 & 5700.200 & -3.997 & & \\
\hline & & & & 5700.205 & -4.094 & & \\
\hline 3247.511 & -1.379 & -0.05 & 2 & 5700.221 & -3.802 & & \\
\hline 3247.513 & -1.028 & & & 5700.227 & -3.647 & & \\
\hline 3247.515 & -1.379 & & & 5700.231 & -3.744 & & \\
\hline 3247.517 & -0.957 & & & 5700.260 & -3.550 & & \\
\hline 3247.520 & -1.426 & & & 5700.266 & -4.152 & & \\
\hline 3247.555 & -0.288 & & & 5700.279 & -3.198 & & \\
\hline 3247.558 & -1.567 & & & 5700.285 & -3.802 & & \\
\hline 3273.927 & -1.375 & -0.35 & 2 & 5782.034 & -3.544 & -1.83 & 4 \\
\hline 3273.929 & -1.024 & & & 5782.042 & -3.845 & & \\
\hline 3273.931 & -2.074 & & & 5782.054 & -3.146 & & \\
\hline 3273.933 & -1.723 & & & 5782.064 & -3.196 & & \\
\hline 3273.971 & -1.024 & & & 5782.073 & -3.497 & & \\
\hline 3273.972 & -1.375 & & & 5782.084 & -2.798 & & \\
\hline 3273.975 & -1.024 & & & 5782.085 & -3.146 & & \\
\hline \multirow[t]{2}{*}{3273.976} & -1.375 & & & 5782.097 & -3.146 & & \\
\hline & & & & 5782.113 & -2.798 & & \\
\hline 5105.504 & -3.720 & -1.51 & 2 & 5782.124 & -2.798 & & \\
\hline 5105.513 & -2.766 & & & 5782.153 & -2.698 & & \\
\hline 5105.516 & -2.813 & & & 5782.172 & -2.350 & & \\
\hline 5105.517 & -3.090 & & & & & & \\
\hline 5105.521 & -2.720 & & & 7933.125 & -0.420 & & 5 \\
\hline 5105.526 & -2.398 & & & & & & \\
\hline 5105.536 & -2.051 & & & 8092.603 & -2.179 & -0.16 & 5 \\
\hline \multirow[t]{2}{*}{5105.563} & -1.942 & & & 8092.604 & -1.831 & & \\
\hline & & & & 8092.612 & -0.971 & & \\
\hline 5153.232 & -0.441 & -0.01 & 2 & 8092.625 & -0.523 & & \\
\hline \multirow[t]{2}{*}{5153.241} & -0.219 & & & 8092.638 & -1.530 & & \\
\hline & & & & 8092.639 & -1.878 & & \\
\hline 5218.200 & -0.934 & 0.27 & 2 & 8092.642 & -1.132 & & \\
\hline 5218.202 & -0.457 & & & 8092.644 & -1.480 & & \\
\hline 5218.206 & -0.236 & & & 8092.651 & -1.132 & & \\
\hline 5218.211 & -0.089 & & & 8092.653 & -1.480 & & \\
\hline 5220.071 & -1.816 & -0.61 & 2 & & & & \\
\hline 5220.073 & -1.338 & & & & & & \\
\hline 5220.077 & -1.117 & & & & & & \\
\hline 5220.082 & -1.131 & & & & & & \\
\hline 5220.083 & -1.481 & & & & & & \\
\hline
\end{tabular}

Notes: References for log gf

1. VALD database Ryabchikova et al. (2015)

2. Kock \& Richter (1968)

3. Kurucz (2014)

4. SUN

5. Carlsson (1988)

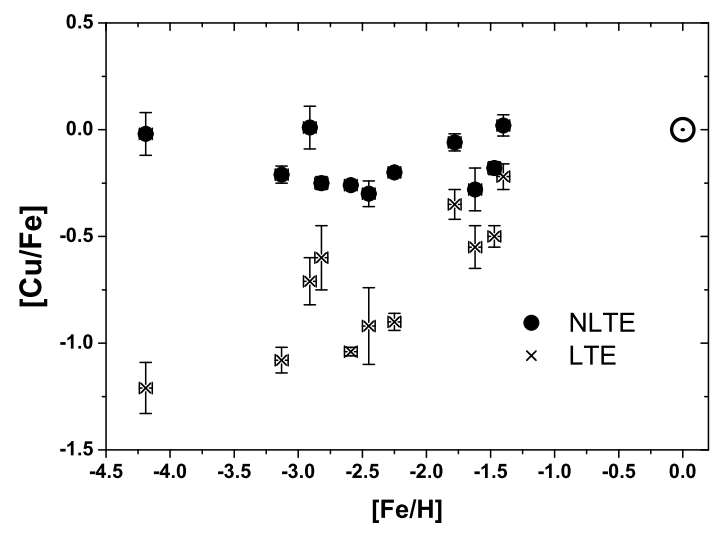

Figure 10. The Galactic evolution of $\mathrm{Cu}$, as captured by our programme stars. Filled symbols are the NLTE results, $\times$ signs are the LTE results.

line is +0.48 dex, while Yan et al. (2016) have a correction that is only +0.17 dex. This is certainly due to the differences between the model atoms used in the two investigations.

The lowest value of $[\mathrm{Cu} / \mathrm{Fe}]$ in our sample is -0.35 for HD 140283, while for the most metal-poor star in our sample, $\mathrm{CD}-38^{\circ} 245$, the $\mathrm{Cu}$ to $\mathrm{Fe}$ ratio is essentially solar, like in CS 31082-001. From our sample we are not in a position to conclude in a robust way if there is a trend of $[\mathrm{Cu} / \mathrm{Fe}]$ with $[\mathrm{Fe} / \mathrm{H}]$ or if there is simply a scatter in $[\mathrm{Cu} / \mathrm{Fe}]$ abudnances.

If we take our $[\mathrm{Cu} / \mathrm{Fe}]$ ratios at face value and take the mean of the sample we find $\langle[\mathrm{Cu} / \mathrm{Fe}]\rangle=-0.15$ with a standard deviation of $0.15 \mathrm{dex}$. The weighted mean is -0.22 dex and the mean error of unit weight (Agekan 1972) is $0.038 \mathrm{dex}$. The fact that this is smaller than the standard deviation suggests that the value of $[\mathrm{Cu} / \mathrm{Fe}]$ is not constant, yet the small size of our sample does not warrant any more detailed statistical analysis. We plan, in the future to apply the NLTE analysis to a much larger sample of stars for which the $\mathrm{Cu}$ resonance lines can be measured. A larger sample may allow to determine in a robust way the Galactic evolution of $\mathrm{Cu}$.

\section{CONCLUSIONS}

Our NLTE model atom allows to derive consistent copper abundances from several atomic lines, over a wide range of stellar parameters, including metallicity. In agreement with previous investigations we confirm that the deviations from NLTE are larger for lower metallicities, yet our NLTE corrections are larger than those that have been found by other groups for the lines in common Shi et al. (2014); Yan et al. $(2015,2016)$. We have been able to show that the UV Cu I resonance lines, that are measurable down to very low metallicities $([\mathrm{Cu} / \mathrm{H}] \leq-4.2)$, can be used as abundance indicators, if treated in NLTE.

The influence of granulation effects on the formation of these lines remains an open issue. Bonifacio et al. (2010) have shown that LTE computations, based on 3D hydrodynamical simulations imply large corrections. Yet those computations were unable to achieve consistency between the UV Cu I resonant doublet and the $5105 \AA$ line. Therefore 
the issue has to be addressed by performing 3D-NLTE computations, that are not currently available.

The picture of the Galactic evolution of $\mathrm{Cu}$ that emerges from our analysis (see Fig. 10) is very different from what has become familiar from previous LTE analysis (Mishenina et al. 2002; Simmerer et al. 2003; Bihain et al. 2004), and that is essentially confirmed by our own LTE analysis, in spite of the limited size of the sample. Our results provide a picture that differs also from the NLTE analysis of Yan et al. (2015). Our small sample is consistent with a mean constant value of $[\mathrm{Cu} / \mathrm{Fe}]=-0.22$ and a scatter of 0.15 dex, in excess of the observational error for the $[\mathrm{Fe} / \mathrm{H}]$ range covered by our sample. A decrease of $[\mathrm{Cu} / \mathrm{Fe}]$ from a nearly solar value at $[\mathrm{Fe} / \mathrm{H}]=-1.5$ down to -0.35 at $[\mathrm{Fe} / \mathrm{H}]=-$ 2.5 , followed by a steep increase to 0.0 cannot be excluded either. A much larger sample is needed to clarify the situation, it is however clear that a NLTE treatment, using our model atom, will result in $[\mathrm{Cu} / \mathrm{Fe}]$ ratios that are considerably larger than those found by other investigations. We can say that, by and large, $\mathrm{Cu}$ tracks closely Fe. This is something that models of Galactic evolution will have to take into account.

\section{ACKNOWLEDGEMENTS}

We are grateful to Ruth Peterson for giving us the reduced HST spectra that have been used in this paper and for useful comments on the manuscript. SMA is thankful to the GEPI Department and Paris Observatoire administration for their hospitality during his visit, and to the Scientific Council of Observatoire de Paris for the financial support. SAK and SMA acknowledge the partial financial support from the SCOPES grant No. IZ73Z0-152485. SAK and AVZh are thankful to the Crimean Council of Ministers for the RFBR grant No. 17-42-92008.

\section{REFERENCES}

Agekan T. A., 1972, Osnovi teorii osibok dla astronomov i fizikov. Izdavateljstvo "Nauka" glavna redakcia fizicko-matematceskog literaturi, Moskva

Allen C. W., 1973, Astrophysical quantities. University of London, Athlone Press

Bihain G., Israelian G., Rebolo R., Bonifacio P., Molaro P., 2004, A\&A, 423, 777

Bisterzo S., Gallino R., Pignatari M., Pompeia L., Cunha K., Smith V., 2004, Mem. Soc. Astron. Italiana, 75, 741

Bonifacio P., Caffau E., Ludwig H.-G., 2010, A\&A, 524, A96

Carlsson M., 1986, Uppsala Astronomical Observatory Reports, 33

Carlsson J., 1988, Phys. Rev. A, 38, 1702

Castelli F., Kurucz R. L., 2003, in Piskunov N., Weiss W. W., Gray D. F., eds, IAU Symposium Vol. 210, Modelling of Stellar Atmospheres. p. A20 (arXiv:astro-ph/0405087)

Cayrel R., et al., 2004, A\&A, 416, 1117

Christlieb N., Gustafsson B., Korn A. J., Barklem P. S., Beers T. C., Bessell M. S., Karlsson T., Mizuno-Wiedner M., 2004, ApJ, 603, 708

Cohen J. G., 1980, ApJ, 241, 981

Cohen J. G., Christlieb N., McWilliam A., Shectman S., Thompson I., Melendez J., Wisotzki L., Reimers D., 2008, ApJ, 672,320

Drawin H.-W., 1968, Zeitschrift fur Physik, 211, 404
Drawin H. W., 1969, Zeitschrift fur Physik, 225, 483

Duffau S., et al., 2017, preprint, (arXiv:1704.02981)

Gerstenberger D. C., Latush E. L., Collins G. J., 1979, Optics Communications, 31, 28

Grevesse N., Scott P., Asplund M., Sauval A. J., 2015, A\&A, 573, A27

Hermann G., Lasnitschka G., Schwabe C., Spengler D., 1993, Spectrochimica Acta, 48, 1259

Kock M., Richter J., 1968, Z. Astrophys., 69, 180

Korotin S. A., Andrievsky S. M., Luck R. E., 1999, A\&A, 351, 168

Kurucz R., 1993, ATLAS9 Stellar Atmosphere Programs and 2 $\mathrm{km} / \mathrm{s}$ grid. Kurucz CD-ROM No. 13. Cambridge, Mass.: Smithsonian Astrophysical Observatory, 1993., 13

Kurucz R. L., 2005a, Memorie della Societa Astronomica Italiana Supplementi, 8, 14

Kurucz R. L., 2005b, Memorie della Societa Astronomica Italiana Supplementi, 8, 86

Kurucz R. L., 2011, Canadian Journal of Physics, 89, 417

Kurucz R. L., 2014, Problems with Atomic and Molecular Data: Including All the Lines. pp 63-73, doi:10.1007/978-3-319-06956-2_6

Kurucz R. L., Furenlid I., Brault J., Testerman L., 1984, Solar flux atlas from 296 to $1300 \mathrm{~nm}$

Lai D. K., Bolte M., Johnson J. A., Lucatello S., Heger A., Woosley S. E., 2008, ApJ, 681, 1524

Liu Y. P., Gao C., Zeng J. L., Yuan J. M., Shi J. R., 2014, ApJS, 211, 30

Lodders K., Palme H., Gail H.-P., 2009, Landolt Börnstein,

Mishenina T. V., Kovtyukh V. V., Soubiran C., Travaglio C., Busso M., 2002, A\&A, 396, 189

Nissen P. E., Schuster W. J., 2011, A\&A, 530, A15

Roederer I. U., et al., 2012, ApJS, 203, 27

Roederer I. U., et al., 2014, ApJ, 791, 32

Ryabchikova T., Piskunov N., Kurucz R. L., Stempels H. C., Heiter U., Pakhomov Y., Barklem P. S., 2015, Phys. Scr., 90, 054005

Sbordone L., 2005, Memorie della Societa Astronomica Italiana Supplementi, 8, 61

Sbordone L., Bonifacio P., Castelli F., Kurucz R. L., 2004, Memorie della Societa Astronomica Italiana Supplementi, 5, 93

Sbordone L., Caffau E., Bonifacio P., Duffau S., 2014, A\&A, 564, A109

Seaton M. J., 1962, in Bates D. R., ed., Atomic and Molecular Processes. New York: Academic Press

Shi J. R., Gehren T., Zeng J. L., Mashonkina L., Zhao G., 2014, ApJ, 782, 80

Simmerer J., Sneden C., Ivans I. I., Kraft R. P., Shetrone M. D., Smith V. V., 2003, AJ, 125, 2018

Siqueira-Mello C., Andrievsky S. M., Barbuy B., Spite M., Spite F., Korotin S. A., 2015, A\&A, 584, A86

Sitnova T., et al., 2015, ApJ, 808, 148

Smiljanic R., et al., 2014, A\&A, 570, A122

Sneden C., Gratton R. G., Crocker D. A., 1991, A\&A, 246, 354

Spite M., Peterson R. C., Gallagher A. J., Barbuy B., Spite F., 2017, A\&A, 600, A26

Steenbock W., Holweger H., 1984, A\&A, 130, 319

Sugar J., Musgrove A., 1990, Journal of Physical and Chemical Reference Data, 19, 527

Summers H. P., 2004, The ADAS User Manual, version 2.6, http://www.adas.ac.uk

Tsymbal V., 1996, in Adelman S. J., Kupka F., Weiss W. W., eds, Astronomical Society of the Pacific Conference Series Vol. 108, M.A.S.S., Model Atmospheres and Spectrum Synthesis. p. 198

Vernazza J. E., Avrett E. H., Loeser R., 1981, ApJS, 45, 635

Yan H. L., Shi J. R., Zhao G., 2015, ApJ, 802, 36

Yan H. L., Shi J. R., Nissen P. E., Zhao G., 2016, A\&A, 585, A102 
Yong D., Meléndez J., Cunha K., Karakas A. I., Norris J. E., Smith V. V., 2008, ApJ, 689, 1020

van Regemorter H., 1962, ApJ, 136, 906

This paper has been typeset from a $\mathrm{T}_{\mathrm{E}} \mathrm{X} / \mathrm{LAT}_{\mathrm{E}} \mathrm{X}$ file prepared by the author. 